\title{
Outage Analysis of EH-based Cooperative NOMA Networks over Generalized Statistical Models
}

\author{
Orken Omarov, Galymzhan Nauryzbayev, ${ }^{\circ}$ Sultangali Arzykulov, ${ }^{\circ}$ Ahmed M. Eltawil, and Mohammad S. Hashmi \\ School of Engineering and Digital Sciences, Nazarbayev University, Nur-Sultan, Kazakhstan \\ ${ }^{\circ}$ Computer, Electrical, and Mathematical Sciences \& Engineering Division, KAUST, Thuwal, KSA 23955-6900 \\ Email: \{orken.omarov, galymzhan.nauryzbayev, mohammad.hashmi\}@nu.edu.kz, \\ ○\{sultangali.arzykulov@gmail.com, ahmed.eltawil@kaust.edu.sa\}
}

\begin{abstract}
In this paper, the outage probability (OP) of the two-hop cooperative non-orthogonal multiple access network with an energy-constrained cooperative agent is evaluated over generalized $\alpha-\mu$ and $\kappa-\mu$ fading models. The power splitting relaying protocol is implemented at the cooperative agent, acting as a relay in amplify-and-forward mode. The effect of hardware impairments (HIs) at the radio frequency front-ends of transceivers are incorporated during the performance evaluation. The results based on the derived analytical expressions suggest the importance of considering HIs for given network architecture and allow one to evaluate the OP over various statistical models.
\end{abstract}

Keywords $-\alpha-\mu, \kappa-\mu$, cooperative communication, nonorthogonal multiple access (NOMA), outage probability, simultaneous wireless information and power transfer (SWIPT).

\section{INTRODUCTION}

The high data rate, low latency and reliability will be an integral part of emerging communication standards [1]. The traffic exchanged wirelessly is forecasted to reach its all-time maximum in recent years [2], whereas the spectrum deficit issue has no final solution yet. The non-orthogonal multiple access (NOMA) is widely recognized to be a key technology to mitigate the frequency shortage [3], through the usage of the same resources like time, frequency or code and the successive interference cancellation (SIC). The SIC is carried out at a weaker receiver node, where the message of a stronger user is decoded and removed first, and then the weaker user decodes its own message [4]. Findings on NOMA suggest its beneficial effect on the improvement of the network's physical performance characteristics [5]. For example, the authors in [6] evaluated the performance of cooperative NOMA network with respect to the conventional one, while the results presented in [7] demonstrated the improved spectral efficiency of NOMA.

The cooperative network has been defined by Nosratinia as the network architecture, where the resources of nodes are intentionally exploited to deliver the message to the destination, through several inter-independent channels, as a result creating a spatial diversity [8]. Some of the applications of NOMA in cooperative networks were considered in [9], [10], where the authors analyzed the improvement of performance metrics. The cooperative network architecture has found its use in applications, where the source-destination link is vulnerable to fading and the node's structure restricts it to have a single antenna for all purposes. Additionally, these applications, such as Internet-of-Things, surveillance and biomedical sensors, often are battery-powered and subject to energy constraints, which can be mitigated through the incorporation of wireless power transfer techniques.
Using the incoming signal for the simultaneous wireless information and energy transfer (SWIPT), thoroughly described in [11], is generally regarded as the prospective solution. One of the widely used practical implementations of the SWIPT for the energy harvesting (EH) purpose is the power splitting relaying (PSR) described in [12]. In [13], the authors presented a performance estimation for energy-constrained two-hop NOMA network with hardware impairments (HIs). However, throughout their work, they assumed that the channels follow the Weibull fading model only. On the other hand, another work in [14] used a generalized $\alpha-\eta-\mu$ fading to model their channels but considered the ideal conditions for their network architecture. Schenk in [15] brought attention to the importance of considering the HIs present at the radiofrequency $(\mathrm{RF})$ front-ends of transceivers, manufactured using the complementary metal-oxide-semiconductor (CMOS) technology, especially when operating with high-frequency signals.

The signal traveling in a wireless medium can be exposed to a variety of channel conditions represented by different statistical distributions. The communication links of mobile nodes in an urban environment, where the message signal is scattered and reflected due to intermediate objects, are often characterized by Rayleigh fading channels [16]. Moreover, in [17], the authors evaluated the performance of the ultra-reliable and low-latency communication architecture for manufacturing plant applications using this fading model. For visible light communications (VLC), the Rician fading model is used to account for the effect of non-line-of-sight and line-of-sight signals at the receiver site. An architecture incorporating the VLC and RF communication is considered in [18], where the authors used the Rician fading model to account for the randomness of the nodes' positions. Furthermore, the authors in [19] suggested the Weibull and Nakagami- $m$ as propagation models for theoretical evaluation of communication performance over $800-900 \mathrm{MHz}$ frequency range. The vehicle-tovehicle communication is modeled using the Weibull model [20], which describes the small-scale fading encountered in an urban environment. Similarly, small-scale Nakagami- $m$ fading is often used to consider multipath clusters in many applications [21]. In order to thoroughly evaluate the network performance, the generalized fading models should be used.

Therefore, this paper evaluates the OP of a two-hop cooperative NOMA architecture over generalized $\alpha-\mu$ and $\kappa-\mu$ fading models. For all the nodes in the architecture, including the wirelessly powered cooperative agent operating in amplify-and-forward (AF) relaying mode, the effects of HIs are considered. The obtained expressions allow assessing the network under a variety of channel fluctuation statistics, such 


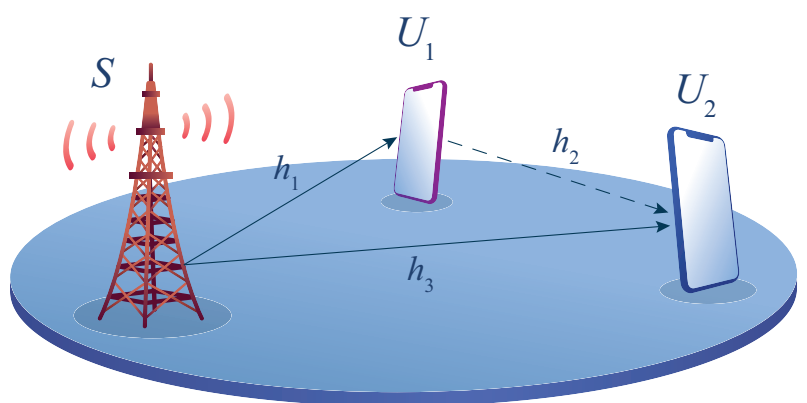

Fig. 1. A two-hop NOMA network with an cooperative agent.

as Nakagami- $m$, Rayleigh, Rice and Weibull. In addition, the effect of hardware imperfections on the OP is determined under different channel fluctuation parameters. Moreover, to acquire some useful insights on the system performance, we provide high SNR asymptotic OP analytical results.

\section{System AND ChANNEL MODELING}

We consider a two-hop NOMA architecture shown in Fig. 1 , where the nodes are equipped with single antennas and operate in a half-duplex mode. The three nodes considered in this system model are the source $(S)$ and two users denoted by $U_{1}$ and $U_{2}$, respectively. The NOMA message of $S, x_{s}=\sum_{i=1}^{2} \sqrt{a_{i} P_{S}} x_{i}$, is broadcasted towards all users, where $i$ and $x_{i}$ denote the users and their messages. The $U_{1}$ acting as a cooperative agent serves as an energy-limited relay operating in the AF mode and harvests its energy from the incoming signal using the PSR protocol. When $U_{1}$ retransmits the NOMA message, the spatial diversity is created at the receiver site of $U_{2}$, as it receives two copies of the same message through mutually independent channels.

For the following network architecture, the channel conditions are assumed to be as $h_{1}>h_{2}$ due to the relative proximity of $U_{1}$ to $S$. Therefore, the power allocation coefficients are set as $a_{1}<a_{2}$, following the power-domain NOMA approach. The channel conditions of source-to-relay ( $S$-to- $\left.U_{1}\right)$, relay-to-destination $\left(U_{1}\right.$-to- $\left.U_{2}\right)$ and source-to-destination $(S$ to- $U_{2}$ ) links are denoted by $h_{1}, h_{2}$ and $h_{3}$, respectively and the distances are symbolized by $d_{1}, d_{2}$ and $d_{3}$. This study describes the channel state fluctuations using the generalized fading models, namely, $\kappa-\mu$ and $\alpha-\mu$. These statistical models are considered as general, because they include other fading models such as Rayleigh, Rice, Nakagami- $m$, Weibull, etc. as special cases [22]-[24]. The probability density function (PDF) of the $\kappa-\mu$ statistical model is given by

$$
f_{h_{i}}(r)=\frac{2 \mu_{i}\left(1+\kappa_{i}\right)^{\frac{\mu_{i}+1}{2}}}{e^{\mu_{i} \kappa_{i}} \kappa_{i}^{\frac{\mu_{i}-1}{2}}} r^{\mu_{i}} e^{-\phi_{i} r^{2}} I_{\mu_{i}-1}\left(2 \mu_{i} \sqrt{\kappa_{i}\left(1+\kappa_{i}\right)} r\right)
$$

where $I_{\nu}(\cdot)$ and $\kappa$ denote the modified Bessel function of the first kind with the order $\nu$ and the power ratio of line-ofsight and scattered signals, accordingly, while $\phi_{i}=\mu_{i}\left(1+\kappa_{i}\right)$. Moreover, the PDF of $\alpha-\mu$ is defined as

$$
f_{h_{i}}(r)=\frac{\alpha_{i} \mu_{i}^{\mu_{i}} r^{\alpha_{i} \mu_{i}-1}}{\hat{r}^{\alpha_{i} \mu_{i}} \Gamma\left(\mu_{i}\right)} \exp \left(-\frac{\mu_{i}}{\hat{r}^{\alpha_{i}}} r^{\alpha_{i}}\right),
$$

where, $\hat{r}=\sqrt[\alpha]{\mathbb{E}\left[r^{\alpha}\right]}, \Gamma(s)=\int_{0}^{\infty} t^{s-1} e^{-t} d t, \alpha$ denote the non-linearity parameter and $\mu$ defines the multipath clusters for both models.

This work considers the effect of residual HIs present at the RF front-ends of transceivers. The combined HIs level of each link, denoted by $\eta_{j \mid j \in\left\{s, u_{1} ; u_{1}, u_{2} ; s, u_{2}\right\}}$, is described through the Complex Gaussian statistic with $\exists_{j}^{2}$ variance and zero mean, with $\ni_{j} \triangleq \sqrt{\vartheta_{j, t}^{2}+\ni_{j, r}^{2}}$, where $t$ and $r$ refer to the transmitter/receiver's HI levels [15].

\section{ENERGY HARVESTING}

In the first time slot, $S$ transmits superimposed message and each user receives the following signal

$$
y_{i}=\sqrt{\frac{P_{S}}{d_{s, u_{i}}^{\tau}}} h_{s, u_{i}}\left(\sum_{j=1}^{2} \sqrt{a_{j}} x_{j}+\eta_{s, u_{i}}\right)+n_{i},
$$

where $\tau$ denotes the path loss factor and $n_{i}$ is the additive white Gaussian noise (AWGN) at $U_{i}$ with $\sigma_{i}^{2}$ variance and zero mean. As the direct path between $S$ and $U_{2}$ exists, the destination node obtains its own message using the signal-tointerference-plus-distortion-plus-noise ratio (SINDR) given as

$$
\gamma_{U_{2}}^{\left\{x_{2}\right\}}=\frac{e_{1} Z}{e_{2} Z+e_{3}},
$$

where $Z=\left|h_{3}\right|^{2}, e_{1}=a_{2}, e_{2}=a_{1}+\vartheta_{s, u_{2}}^{2}$, and $e_{3}=\frac{\sigma^{2} d_{3}^{\tau}}{P_{s}}$.

In the PSR protocol, the whole transmission block denoted by $T$ is divided into equal two time slots. During the first time slot, $U_{1}$ uses the amount of power $\rho P_{S}$ from the incoming signal for the energy harvesting and the remaining $(1-\rho) P_{S}$ portion of it is dedicated for information detection purposes. Whereas, during the second time slot, $U_{1}$ uses the harvested energy to enable the $U_{1}-U_{2}$ communication. Thus, the corresponding harvested energy at $U_{1}$ can be given as

$$
E^{H}=0.5 \varpi \rho T\left(P_{S}\left|h_{1}\right|^{2}\left(1+\vartheta_{s, u_{1}}^{2}\right) d_{1}^{-\tau}+\sigma_{1}^{2}\right),
$$

where $0 \leq \varpi \leq 1$ is the energy conversion efficiency. Neglecting the energy harvested from the noise, the power to be exploited during the next time slot is defined as

$$
P^{H}=2 E^{H} / T=\varpi \rho P_{S} d_{1}^{-\tau}\left(1+\vartheta_{s, u_{1}}^{2}\right)\left|h_{1}\right|^{2} .
$$

For the information detection, $y_{1}^{I T}=\sqrt{1-\rho} y_{1}+n_{c}$ is used, where $n_{c}$ denotes the AWGN of the receiver.

$U_{1}$ decodes the message $x_{2}$ first, using the successive interference cancellation (SIC), and $x_{1}$ second, with signalto-distortion-plus-noise ratio (SDNR) of

$$
\gamma_{U_{1}}^{\left\{x_{1}\right\}}=\frac{b_{1} X}{b_{2} X+b_{3}}
$$

where $X=\left|h_{1}\right|^{2}, \quad b_{1}=a_{1}, b_{2}=\ni_{s, u_{1}}^{2}$ and $b_{3}=$ $\frac{d_{1}^{\tau}}{P_{S}}\left(\sigma_{a}^{2}+\frac{\sigma_{c}^{2}}{1-\rho}\right)$. Further, $U_{1}$ forwards the message using the accumulated energy during the last time slot and the message received by $U_{2}$ during this time slot is represented as

$$
y_{1 \rightarrow 2}=\frac{G h_{2}}{\sqrt{d_{2}^{\tau}}}\left(\sqrt{1-\rho} y_{1}+n_{c}+\eta_{u_{1}, u_{2}}\right)+n_{2},
$$


where $G$ is the relay gain. Applying the high SNR approximation, the relay gain expression becomes

$G=\sqrt{\frac{\varpi \rho P_{s}\left(1+\vartheta_{s, u_{1}}^{2}\right)\left|h_{1}\right|^{2}}{(1-\rho)\left(P_{s}\left(1+\vartheta_{s, u_{1}}^{2}\right)\left|h_{1}\right|^{2}+d_{1}^{\tau} \sigma_{1}^{2}\right)+d_{1}^{\tau} \sigma_{c}^{2}}} \simeq \sqrt{\frac{\varpi \rho}{1-\rho}}$.

By substitution, the SINDR of $U_{2}$ for $x_{2}$ is defined as

$$
\gamma_{U_{1 \rightarrow 2}}^{\left\{x_{2}\right\}}=\frac{c_{1} X Y}{c_{2} X Y+c_{3} Y+c_{4}}
$$

where $Y=\left|h_{2}\right|^{2}, c_{1}=a_{2}, c_{2}=a_{1}+\ni_{s, u_{1}}^{2}, c_{3}=$ $\frac{d_{1}^{\tau}}{P_{S}}\left(\sigma_{a}^{2}+\frac{\sigma_{c}^{2}+\vartheta_{u_{1}, u_{2}}^{2}}{(1-\rho)}\right)$ and $c_{4}=\frac{d_{1}^{\tau} d_{2}^{\tau} \sigma_{2}^{2}}{\varpi \rho P_{S}}$.

\section{Outage Performance}

The outage at $U_{i}$ occurs when the achievable transmission rate of this user falls behind the threshold, denoted by $\mathcal{I}_{\mathrm{th}, i}$, and is defined as

$$
P_{\text {out }, i}=\operatorname{Pr}\left(\frac{1}{2} \log _{2}\left(1+\gamma_{U_{i}}\right)<\mathcal{I}_{\mathrm{th}, i}\right),
$$

where $\gamma_{U_{i}}$ is the effective SNR at user $U_{i}$.

The outage performance of $U_{2}$ can be defined as following, where maximum ratio combining (MRC) is used to incorporate cooperation of direct link and the signal amplified via relay

$$
P_{\text {out }, 2}=\operatorname{Pr}\left(\gamma_{U_{2}}+\gamma_{U_{1 \rightarrow 2}}<v_{2}\right) .
$$

where $v_{i}=2^{2 \mathcal{I}_{\mathrm{th}, i}}-1$ is the predefined rate threshold. Using the inequality $\min (a, b)<(a+b) / 2$, a lower bound of the inner expression of (14) can be rewritten as

$$
\begin{aligned}
P_{\text {out }, 2} & =\operatorname{Pr}\left(\min \left(\gamma_{U_{2}}, \gamma_{U_{1 \rightarrow 2}}\right)<\frac{v_{2}}{2}\right) \\
& =1-\operatorname{Pr}\left(\gamma_{U_{2}}>\frac{v_{2}}{2}\right) \operatorname{Pr}\left(\gamma_{U_{1 \rightarrow 2}}>\frac{v_{2}}{2}\right) \\
& =1-\left(1-D_{1}\right)\left(1-D_{2}\right)=D_{1}+D_{2}-D_{1} D_{2},
\end{aligned}
$$

where $D_{1}=\operatorname{Pr}\left(\gamma_{U_{2}}^{\text {direct }}<0.5 v_{2}\right)$ and $D_{2}=$ $\operatorname{Pr}\left(\gamma_{U_{1 \rightarrow 2}}<0.5 v_{2}\right)$. Thus, the followings are true

$$
\begin{aligned}
D_{1} & =\operatorname{Pr}\left(\gamma_{U_{2}}<0.5 v_{2}\right)=\operatorname{Pr}\left(Z<\frac{0.5 v_{2} e_{3}}{e_{1}-0.5 v_{2} e_{2}}\right) \\
& =\int_{0}^{\frac{0.5 v_{2} e_{3}}{e_{1}-0.5 v_{2} e_{2}}} f_{Z}(z) \mathrm{d} z \\
D_{2} & =\operatorname{Pr}\left(\gamma_{U_{1 \rightarrow 2}}<0.5 v_{2}\right)=\operatorname{Pr}\left(X<\frac{p_{1}}{Y}+p_{2}\right), \\
& =\int_{0}^{\infty} f_{Y}(y) \int_{0}^{\frac{p_{1}}{y}+p_{2}} f_{X}(x) \mathrm{d} x \mathrm{~d} y,
\end{aligned}
$$

where $p_{1}=\frac{0.5 v_{2} c_{4}}{\left(c_{1}-0.5 v_{2} c_{2}\right)}$ and $p_{2}=\frac{0.5 v_{2} c_{3}}{\left(c_{1}-0.5 v_{2} c_{2}\right)}$.

Proposition 1: For the $\alpha-\mu$ distribution, the OP expression is defined as in (11), at the top of the next page, where $E=\lambda_{3}\left(\frac{0.5 v_{2} e_{3}}{e_{1}-0.5 v_{2} e_{2}}\right)^{\frac{\alpha_{3}}{2}}, B=\frac{\alpha_{2} \lambda_{2}^{\mu_{2}}}{2 \Gamma\left(\mu_{2}\right)}, C=\frac{\frac{\alpha_{1} m}{2} !}{\left(\frac{\alpha_{1} m}{2}-r\right) ! r !}$, $A=\frac{\lambda_{1} \alpha_{1} p_{1}}{p_{2}^{1-\frac{\alpha_{1}}{2}} \alpha_{2}}$, and $F=\frac{e^{-\lambda_{1} p_{2}^{\frac{\alpha_{1}}{2}}} \sqrt{\frac{2}{\alpha_{2}}}}{(2 \pi)^{\frac{\alpha_{2}}{4}-0.5}}$.

Proof: See Appendix A.
Proposition 2: For the $\kappa-\mu$ distribution, the OP expression is defined as in (12), at the top of the next page, where $J=$

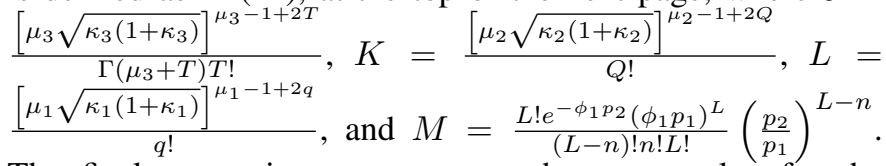
The final expression converges to the exact value for the summation of first 20 terms for variables $T_{\infty}, Q_{\infty}$, and $q_{\infty}$, i.e., $T_{\infty}=Q_{\infty}=q_{\infty}=20$.

Proof: See Appendix B.

\section{Numerical Results}

This section illustrates the analytical and simulation results for the performance evaluation of the considered network architecture under different $\alpha, \kappa$ and $\mu$ parameters and a set of HIs levels. Unless stated otherwise, the system parameters for the rest of the paper are set as: $d_{1}=2 \mathrm{~m}^{1}, d_{2}=1.3 \mathrm{~m}, d_{3}=3$ $\mathrm{m}, \tau=3, \varpi=0.8, \rho=0.9, v=3, a_{1}=0.2$ and $a_{2}=0.8$. Some fading models such as Rayleigh and Nakagami- $m$ can be implemented using both $\alpha-\mu$ and $\kappa-\mu$ models. Whereas, Weibull and Rice can only be implemented using $\alpha-\mu$ and $\kappa$ $\mu$ models, respectively. According to [25], the observed values of $\ni$ range between 0.08 and 0.175 . For comparison purposes, its range is $\left[\begin{array}{ll}0 & 0.2\end{array}\right]$ in our simulations.

Fig. 2 demonstrates the change of the OP under the ideal and imperfect hardware conditions for both generalized fading models. The analytical results for both fading models coincide with each other where it is applicable, and match with the Monte-Carlo simulation results. Such results support the correctness of the derived OP expressions. Overall, the performance degradation is observed for all combinations of channel conditions when increasing the HIs level. Interestingly, the results reveal that the effect of HIs is more severe at higher values of $\alpha-\mu$ and $\kappa-\mu$ parameters. These results revealed a stronger correlation between the improvement of outage probability and the increase in the value of $\mu$ rather than $\kappa$.

Similarly, Fig. 3 illustrates the OP over Rice, Weibull and Nakagami- $m$ fading models for NOMA and OMA scenarios at $\ni=0.2$. The proposed network architecture delivers the messages to their respective destination nodes in two time-slots, while the OMA enabled network requires three time slots. Fig. 3 shows a considerable difference between the NOMA and OMA OP results over all of the statistical models, with OMA generally having worse OP characteristics. Additionally, it can be seen that the high SNR approximation plots coincide with OP results. The OP results for Rice $(\kappa=3, \mu=1)$ and Nakagami- $m(\kappa=0, \mu=3)$ channels support the claim that the effect of increasing parameter $\mu$ has a more significant impact on the OP improvement rather than $\kappa$.

Fig. 4 demonstrates the relationship of the OP with predefined rate threshold at $38 \mathrm{~dB}$ transmit SNR, for special cases of the generalized $\alpha-\mu$ fading model. The increase in HIs level is observed to have a more drastic effect on the OP at Nakagami$m(\alpha=2, \mu=4)$ rather than at Rayleigh $(\alpha=2, \mu=1)$ or even Weibull ( $\alpha=4, \mu=1)$ cases. For the purpose of being explicit, the value of parameter $\mu$ used in Nakagami- $m$ model

\footnotetext{
${ }^{1}$ We assume that the QoS of $U_{1}$ will be satisfied due to its proximity to $S$.
} 


$$
P_{\text {out }, 2}^{\alpha-\mu}=1-\left[1-\frac{\gamma_{i}\left(\mu_{3}, E\right)}{\Gamma\left(\mu_{3}\right)}\right]\left[B \sum_{m=0}^{\mu_{1}-1} \frac{\lambda_{1}^{m} p_{1}^{\frac{\alpha_{1} m}{2}}}{m !} \sum_{r=0}^{\alpha_{1} m / 2} C\left(\frac{p_{2}}{p_{1}}\right)^{\frac{\alpha_{1} m}{2}-r} F A^{\frac{\alpha_{2} \mu_{2}}{2}-r} G_{0, \quad \frac{\alpha_{2}}{2}+1, \quad 0}^{\frac{\alpha_{2}}{2}+1}\left(\lambda_{2} A^{\frac{\alpha_{2}}{2}} \mid \begin{array}{l}
0, \Delta\left(\frac{\alpha_{2}}{2}, r-\frac{\alpha_{2} \mu_{2}}{2}\right)
\end{array}\right)\right]
$$

$$
\begin{aligned}
P_{\text {out }, 2}^{\kappa-\mu}= & 1-\left(1-\sum_{T=0}^{T_{\infty}} \psi_{3} J \phi_{3}^{-\left(\mu_{3}+T\right)} \gamma_{i}\left(\mu_{3}+T, \phi_{3}\left(\frac{0.5 v_{2} e_{3}}{e_{1}-0.5 v_{2} e_{2}}\right)\right)\right)\left(1-\left[\sum_{Q=0}^{Q_{\infty}} \sum_{q=0}^{q_{\infty}} \psi_{2} K \psi_{1} L \phi_{1}^{-\left(\mu_{1}+q\right)} \phi_{2}^{-\left(\mu_{2}+Q\right)}\right]\right. \\
& \left.+\left[\sum_{Q=0}^{Q_{\infty}} \sum_{q=0}^{q_{\infty}} \sum_{L=0}^{\mu_{1}+q-1} \sum_{n=0}^{L} \frac{\psi_{2} K \psi_{1} L}{\Gamma\left(\mu_{2}+Q\right)} \phi_{1}^{-\left(\mu_{1}+q\right)} M \phi_{2}^{-\left(\mu_{2}+Q-n\right)} G_{2,0}^{0,2}\left(\frac{1}{\phi_{1} \phi_{2} p_{1}} \mid \begin{array}{cc}
1, & 1-\left(\mu_{2}+Q-n\right) \\
-, & -
\end{array}\right)\right]\right)
\end{aligned}
$$

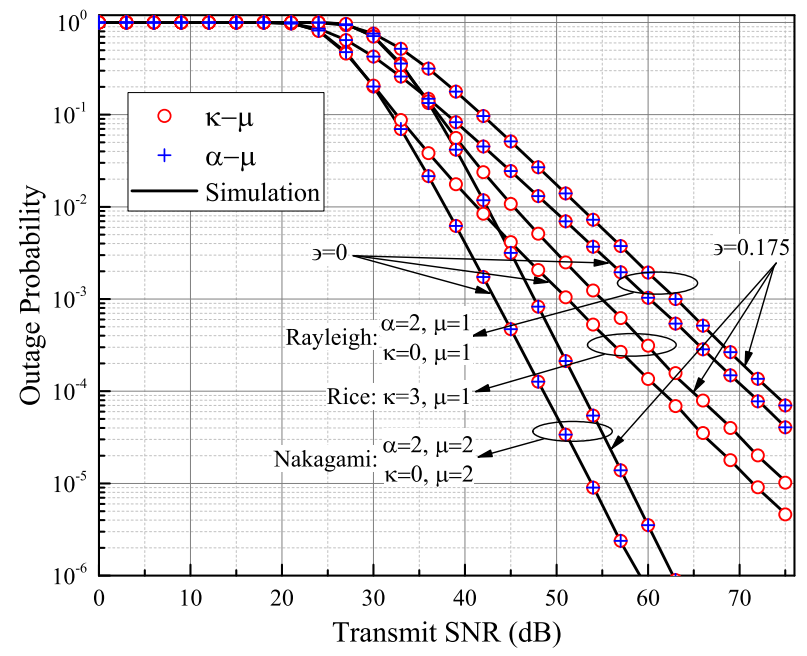

Fig. 2. The outage probability vs. the transmit SNR when $\ni$ $=\{0,0.175\}$.

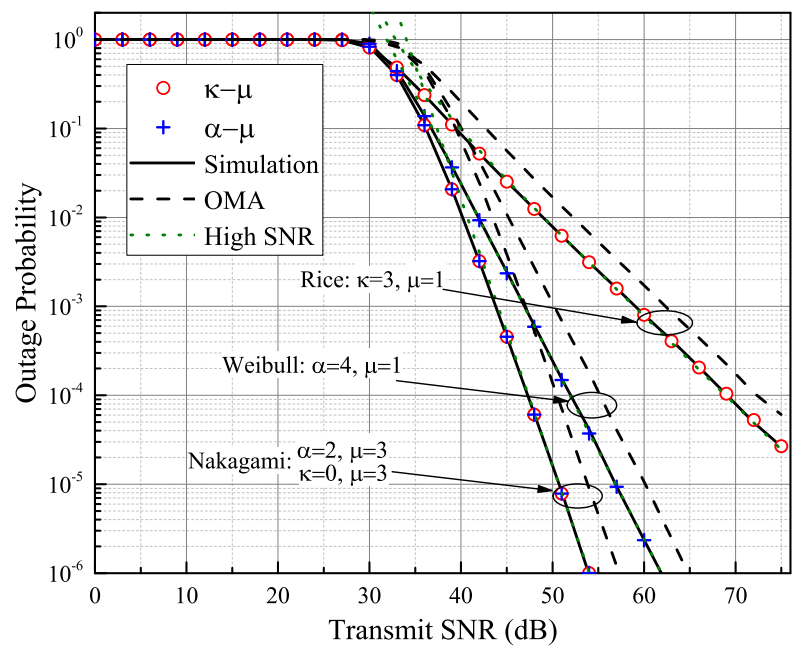

Fig. 3. The outage probability vs. the transmit SNR for NOMA, OMA and high SNR scenarios when $\ni=0.2$.

is intentionally chosen to have different value than in previous graphs. Overall, the OP metric degrades with increase in the value of the predefined SNR threshold, where the full outage can be observed over all statistical models at $8 \mathrm{~dB}$.

Fig. 5 illustrates the average throughput versus the transmit SNR at $v=2$. In the same way as the OP, the throughput

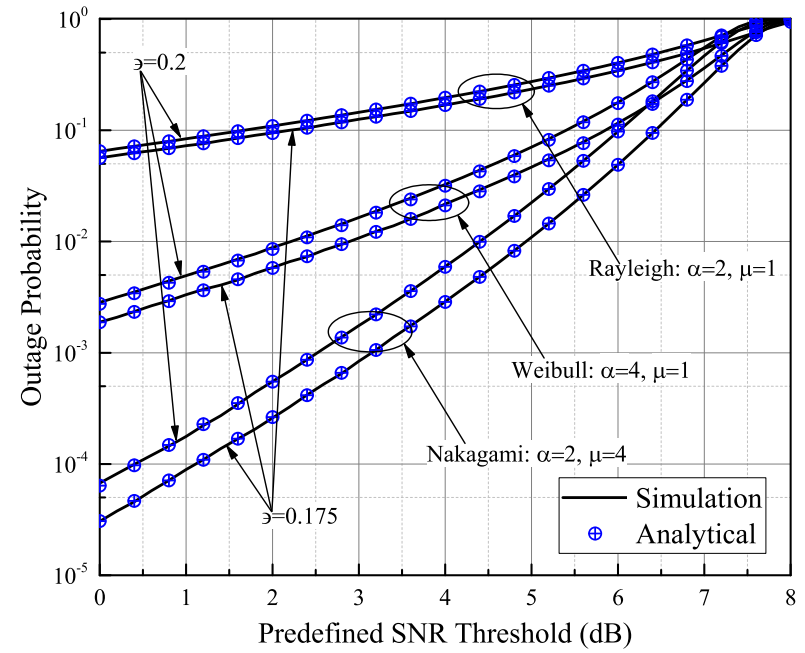

Fig. 4. The outage probability vs. the SNR threshold at $38 \mathrm{~dB}$ when $\ni=\{0.175,0.2\}$.

degrades with an increase in $\ni$ values. Due to the network architecture and the NOMA technique, the throughput values saturate for all observed cases. For comparison purposes, the values of $\ni=\{0,0.175\}$ are considered to evaluate the throughput over Nakagami- $m(\kappa=0, \mu=3)$, Rayleigh $(\kappa=0, \mu=1)$ and Rice $(\kappa=1, \mu=1)$ fading models. The results suggest that increasing the number of multipath clusters, $\mu$, enhances the average throughput. The minimum throughput of around $2.16 \mathrm{bps} / \mathrm{Hz}$ is observed at a maximum value of HIs, $\ni=0.175$. At the same time, the throughput of an ideal hardware case saturates at around $2.32 \mathrm{bps} / \mathrm{Hz}$.

\section{CONCLUSION}

This study contributes to the area of performance analysis of hardware-limited SWIPT-enabled cooperative NOMA networks, through the derivation of expressions describing the OP metric over generalized $\alpha-\mu$ and $\kappa-\mu$ fading models. These models include the Rice, Nakagami- $m$, Rayleigh and Weibull statistical models as their special cases. The analytical expressions were analysed over a set of HIs levels and exhibited the consistency over $\alpha-\mu$ and $\kappa-\mu$ models, and were further validated by Monte-Carlo simulations. The results of this study underline the importance of considering the HIs present at $\mathrm{RF}$ front-ends of transceivers, when applications with strict reliability conditions considered. Especially, the results suggest that the effect of HIs on the OP enhances with the increase in 


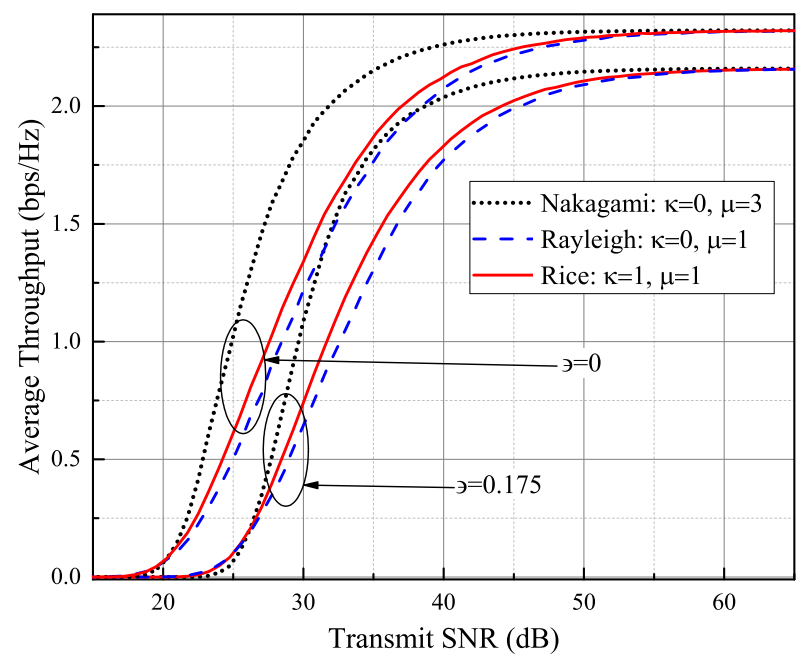

Fig. 5. The average throughput vs. the transmit SNR when 7 $=\{0,0.175\}$.

the value of $\alpha, \kappa$ and $\mu$ parameters.

\section{ACKNOWLEDGMENT}

This work was supported by the Nazarbayev University Faculty Development Competitive Research Program under Grant no. 240919FD3935.

\section{APPENDIX A}

DERIVATION OF OUTAGE PROBABILITY FOR $\alpha-\mu$ FADING

Using the "change of variable" method for $X=\left|h_{i}\right|^{2}$, we can write the PDF of a new random variable as

$$
f_{X}(x)=\frac{\alpha_{1} \lambda_{1}^{\mu_{1}}}{2 \Gamma\left(\mu_{1}\right)} x^{\frac{\alpha_{1} \mu_{1}}{2}-1} \exp \left(-\lambda_{1} x^{\frac{\alpha_{1}}{2}}\right)
$$

where $\lambda_{i}=\frac{\mu_{i}}{\hat{r}_{i}}$. The same is true for $Z$ and $Y$.

Now, $D_{1}$ in (16) can be further rewritten as

$$
D_{1}=\int_{0}^{\frac{0.5 v_{2} e_{3}}{e_{1}-0.5 v_{2} e_{2}}} f_{Z}(z) \mathrm{d} z=\frac{\gamma_{i}\left(\mu_{3}, E\right)}{\Gamma\left(\mu_{3}\right)},
$$

where the lower incomplete gamma function is defined as $\gamma_{i}(a, x)=\int_{0}^{x} t^{a-1} e^{-t} \mathrm{~d} t$. Similarly, $D_{2}$ in (17) is written as

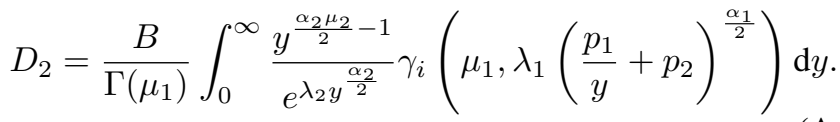

Then, by using the lower incomplete gamma function series expansion as $\gamma_{i}(a, x)=\Gamma(a)\left(1-e^{-x} \sum_{L=0}^{a-1} \frac{x^{L}}{L !}\right)$, as well as the Binomial expansion theorem, $D_{2}$ can be expressed as

$$
\begin{aligned}
D_{2}= & 1-\left[B \sum_{m=0}^{\mu_{1}-1} \frac{\lambda_{1}^{m} p_{1}^{\frac{\alpha_{1} m}{2}}}{m !} \sum_{r=0}^{\frac{\alpha_{1} m}{2}} C\left(\frac{p_{2}}{p_{1}}\right)^{\frac{\alpha_{1} m}{2}-r}\right. \\
& \left.\times \int_{0}^{\infty} y^{\frac{\alpha_{2} \mu_{2}}{2}-r-1} e^{-\lambda_{2} y^{\frac{\alpha_{2}}{2}}} e^{-\lambda_{1}\left(\frac{p_{1}}{y}+p_{2}\right)^{\frac{\alpha_{1}}{2}}} \mathrm{~d} y\right] .
\end{aligned}
$$

Furthermore, applying the binomial approximation of

$$
\left(1+\frac{p_{1}}{p_{2}} y^{-1}\right)^{\frac{\alpha_{1}}{2}} \approx 1+\frac{\alpha_{1}}{2} \frac{p_{1}}{p_{2}} y^{-1}
$$

and the following representations

$$
\begin{aligned}
e^{-\lambda_{2} y^{\frac{\alpha_{2}}{2}}} & =G_{0,1}^{1,0}\left(\lambda_{2} y^{\frac{\alpha_{2}}{2}} \mid-\frac{-}{0}\right), \\
e^{-\frac{\lambda_{1} p_{2}^{\frac{\alpha_{1}}{2}-1} \alpha_{1} p_{1}}{2 y}} & =G_{1,0}^{0,1}\left(\frac{2 y}{\lambda_{1} p_{2}^{\frac{\alpha_{1}}{2}-1} \alpha_{1} p_{1}} \mid \frac{1}{-}\right),
\end{aligned}
$$

$D_{2}$ is solved using [26, Eq. (21)].

\section{A. High-SNR Approximation}

Assuming the high SNR, the expression $\lambda_{1}\left(p_{1}\left(y^{-1}+\frac{p_{2}}{p_{1}}\right)\right)^{\frac{\alpha_{1}}{2}}$ converges to zero, where $\frac{p_{2}}{p_{1}}$ is a constant and $p_{1} \rightarrow 0$. Using the binomial expansion theorem and taking into account $\gamma_{i}(c, d) \approx \frac{d^{c}}{c}$, when $d \rightarrow 0$, the expression (A.3) can be written as

$$
D_{2}=\frac{\lambda_{1}^{\mu_{1}} p_{1}^{R}}{\Gamma\left(\mu_{1}\right) \Gamma\left(\mu_{2}\right) \mu_{1}} \sum_{r=0}^{R} H\left(\frac{p_{2}}{p_{1}}\right)^{R-r} \lambda_{2}^{\frac{2 r}{\alpha_{2}}} \Gamma\left(\mu_{2}-\frac{2 r}{\alpha_{2}}\right)
$$

where $H=\frac{\frac{\alpha_{1} \mu_{1}}{2} !}{\left(\frac{\alpha_{1} \mu_{1}}{2}-r\right) ! r !}$ and $R=\frac{\alpha_{1} \mu_{1}}{2}$. Similarly, applying the high-SNR approximation to (A.2) results in $D_{1}=\frac{E^{\mu_{3}}}{\mu_{3} \Gamma\left(\mu_{3}\right)}$. Finally, substituting these expressions into (15), the high-SNR approximation for the $\alpha-\mu$ fading model is derived as in (A.8).

\section{APPENDIX B}

\section{DERIVATION OF OUTAGE PROBABILITY FOR $\kappa-\mu$ FADING}

Using the "change of variable" method for $Z=\left|h_{3}\right|^{2}$, and defining $\psi=\frac{\mu(1+\kappa)^{\frac{\mu+1}{2}}}{e^{\mu \kappa} \kappa^{\frac{\mu-1}{2}}}$, the PDF of $Z$ can be written as

$$
f_{Z}(z)=\psi z^{\frac{\mu-1}{2}} e^{-\phi z} I_{\mu-1}(2 \mu \sqrt{\kappa(1+\kappa) z}) .
$$

Representing the modified Bessel function of the first kind as

$$
I_{\mu-1}(2 \mu \sqrt{\kappa(1+\kappa) z})=\sum_{q=0}^{q_{\infty}} \frac{[\mu \sqrt{\kappa(1+\kappa) z}]^{\mu-1+2 q}}{\Gamma(\mu+q) q !},
$$

$D_{1}$ is given by

$$
D_{1}=\sum_{T=0}^{T_{\infty}} \psi_{3} J \phi_{3}^{-\left(\mu_{3}+T\right)} \gamma_{i}\left(\mu_{3}+T, \phi_{3}\left(\frac{0.5 v_{2} e_{3}}{e_{1}-0.5 v_{2} e_{2}}\right)\right) \text {. }
$$

Using the series representation of lower incomplete gamma function and applying [26, Eq. (21)], $D_{2}$ can be found as

$$
\begin{aligned}
D_{2} & =\left[\sum_{Q=0}^{Q_{\infty}} \sum_{q=0}^{q_{\infty}} \psi_{2} K \psi_{1} L \phi_{1}^{-\left(\mu_{1}+q\right)} \phi_{2}^{-\left(\mu_{2}+Q\right)}\right] \\
& -\left[\sum_{Q=0}^{Q_{\infty}} \sum_{q=0}^{q_{\infty}} \sum_{L=0}^{\mu_{1}+q-1} \sum_{n=0}^{L} \frac{\psi_{2} K \psi_{1} L}{\Gamma\left(\mu_{2}+Q\right)} \phi_{1}^{-\left(\mu_{1}+q\right)} M \phi_{2}^{-\left(\mu_{2}+Q-n\right)}\right.
\end{aligned}
$$




$$
\begin{aligned}
P_{\mathrm{out}, \mathrm{HS}}^{\kappa-\mu}=1-\left(1-\sum_{T=0}^{\infty} \psi_{3} J \phi_{3}^{-\left(\mu_{3}+T\right)} \frac{\left(\frac{0.5 v_{2} e_{3} \phi_{3}}{e_{1}-0.5 v_{2} e_{2}}\right)^{\mu_{3}+T}}{\mu_{3}+T}\right) & \left(1-\left[\sum_{Q=0}^{\infty} \sum_{q=0}^{\infty} \frac{\psi_{2} K \psi_{1} L p_{2}^{\mu_{1}+q}}{\Gamma\left(\mu_{1}+q\right)} \frac{\phi_{2}^{-\left(\mu_{2}+Q\right)}}{\mu_{1}+q}\right]\right. \\
+ & {\left.\left[\sum_{Q=0}^{\infty} \sum_{q=0}^{\infty} \frac{\psi_{2} K \psi_{1} L p_{2}^{\mu_{1}+q-1} \phi_{2}^{-\left(\mu_{2}+Q\right)}}{\Gamma\left(\mu_{1}+q\right) \Gamma\left(\mu_{2}+Q\right)} p_{1} \Gamma\left(\mu_{1}+q-1\right)\right]\right) }
\end{aligned}
$$

$$
\begin{array}{r}
P_{\text {out }, \mathrm{HS}}^{\alpha-\mu}=1-\left[1-\frac{E^{\mu_{3}}}{\mu_{3} \Gamma\left(\mu_{3}\right)}\right]\left[1-\frac{\lambda_{1}^{\mu_{1}} p_{1}}{\Gamma\left(\mu_{1}\right) \Gamma\left(\mu_{2}\right.}\right. \\
\times G_{2,0}^{0,2}\left(\frac{1}{\phi_{1} \phi_{2} p_{1}} \mid \begin{array}{cc}
1, & 1-\left(\mu_{2}+Q-n\right) \\
-, & -
\end{array}\right] . \text { (B.4) }
\end{array}
$$

\section{A. High-SNR Approximation}

Assuming the high SNR condition (when $e_{3} \rightarrow 0$ ), $D_{1}$ in (16) can be rewritten as

$$
D_{1}=\sum_{T=0}^{T_{\infty}} \psi_{3} J \phi_{3}^{-\left(\mu_{3}+T\right)} \frac{\left(\frac{0.5 v_{2} e_{3} \phi_{3}}{e_{1}-0.5 v_{2} e_{2}}\right)^{\mu_{3}+T}}{\mu_{3}+T} .
$$

Using the binomial approximation, $D_{2}$ in (17) is expressed as

$$
\begin{aligned}
D_{2} & =\sum_{Q=0}^{Q_{\infty}} \sum_{q=0}^{q_{\infty}} \frac{\psi_{2} K \psi_{1} L p_{2}^{\mu_{1}+q}}{\Gamma\left(\mu_{1}+q\right)} \frac{\phi_{2}^{-\left(\mu_{2}+Q\right)}}{\mu_{1}+q} \\
& -\sum_{Q=0}^{Q_{\infty}} \sum_{q=0}^{q_{\infty}} \frac{\psi_{2} K \psi_{1} L p_{2}^{\mu_{1}+q-1} \phi_{2}^{-\left(\mu_{2}+Q\right)}}{\Gamma\left(\mu_{1}+q\right) \Gamma\left(\mu_{2}+Q\right)} p_{1} \Gamma\left(\mu_{1}+q-1\right) .
\end{aligned}
$$

Finally, substituting these expressions into (15), the high-SNR approximation for the $\kappa-\mu$ fading model is derived as in (B.7).

\section{REFERENCES}

[1] L. U. Khan et al., "6G wireless systems: A vision, architectural elements, and future directions," IEEE Access, vol. 8, pp. 147029-147044, Aug. 2020.

[2] Cisco, "Annual Internet Report 2018-2023 (white paper)," CISCO, Tech. Rep., Updated on Mar. 2020.

[3] S. Arzykulov et al., "Underlay Spectrum Sharing for NOMA Relaying Networks: Outage Analysis," Int. Conf. Comput., Netw. Commun. (ICNC), Big Island, HI, USA, 2020, pp. 897-901.

[4] Z. Ding et al., "A Survey on Non-Orthogonal Multiple Access for 5G Networks: Research Challenges and Future Trends," IEEE J. Sel. Areas Commun., vol. 35, no. 10, pp. 2181-2195, Oct. 2017.

[5] Z. Ding et al., "On the Performance of Non-Orthogonal Multiple Access in 5G Systems with Randomly Deployed Users," IEEE Signal Process. Lett., vol. 21, no. 12, pp. 1501-1505, Dec. 2014.

[6] X. Liang et al., "Outage performance for cooperative NOMA transmission with an AF relay," IEEE Commun. Lett., vol. 21, no. 11, pp. 2428-2431, Nov. 2017.

[7] J. Men et al., "Performance analysis of non-orthogonal multiple access for relaying networks over Nakagami- $m$ fading channels," IEEE Trans. Veh. Technol. vol. 66, no. 2, pp. 1200-1208, Feb. 2017.

[8] A. Nosratinia et al., "Cooperative communication in wireless networks," IEEE Commun. Mag., vol. 42, no. 10, pp. 74-80, Oct. 2004.
[9] Z. Ding et al., "Cooperative Non-Orthogonal Multiple Access in 5G Systems," IEEE Commun. Lett., vol. 19, no. 8, pp. 1462-1465, Aug. 2015.

[10] Y. Liu et al., "Cooperative non-orthogonal multiple access with simultaneous wireless information and power transfer," IEEE J. Sel. Areas Commun., vol. 34, no. 4, pp. pp. 938-953, Apr. 2016.

[11] L. R. Varshney, "Transporting information and energy simultaneously," IEEE Int. Symp. Inf. Theory, Toronto, ON, 2008, pp. 1612-1616.

[12] A. A. Nasir et al., "Relaying Protocols for Wireless Energy Harvesting and Information Processing," IEEE Trans. Wirel. Commun., vol. 12, no. 7, pp. 3622-3636, Jul. 2013.

[13] X. Li et al., "Performance Analysis of Impaired SWIPT NOMA Relaying Networks Over Imperfect Weibull Channels," IEEE Systems J., vol. 14, no. 1, pp. 669-672, Mar. 2020.

[14] A.S. Alqahtani and E. Alsusa, "Performance Analysis of Downlink NOMA System over $\alpha-\eta-\mu$ Generalized Fading Channel," IEEE Veh. Technol. Conf. (VTC2020-Spring), Antwerp, Belgium, 2020, pp. 1-5.

[15] T. Schenk, RF Imperfections in High-rate Wireless Systems: Impact and Digital Compensation. Dordrecht, The Netherlands: Springer, 2008.

[16] A. S. Akki and F. Haber, "A statistical model of mobile-to-mobile land communication channel," IEEE Trans. Veh. Technol., vol. 35, no. 1, pp. 2-7, Feb. 1986.

[17] N. A. Johansson et al., "Radio access for ultra-reliable and lowlatency 5G communications," 2015 IEEE Int. Conf. Commun. Workshop (ICCW), pp. 1184-1189, London, 2015.

[18] G. Pan, J. Ye, and Z. Ding, "Secure Hybrid VLC-RF Systems With Light Energy Harvesting," IEEE Trans. Commun., vol. 65, no. 10, pp. 4348-4359, Oct. 2017.

[19] "Coverage prediction for mobile radio systems operating in the 800/900 MHz frequency range," IEEE Trans. Veh. Technol., vol. 37, no. 1, pp. 3-72, Feb. 1988.

[20] Q. Wu, D. W. Matolak, and I. Sen, "5-GHz-Band Vehicle-to-Vehicle Channels: Models for Multiple Values of Channel Bandwidth," IEEE Trans. Veh. Technol., vol. 59, no. 5, pp. 2620-2625, June 2010.

[21] L. Lv et al., "Application of Non-Orthogonal Multiple Access in Cooperative Spectrum-Sharing Networks Over Nakagami- $m$ Fading Channels," IEEE Trans. Veh. Technol., vol. 66, no. 6, pp. 5506-5511, June 2017.

[22] M. D. Yacoub, "The $\kappa-\mu$ distribution and the $\eta-\mu$ distribution," IEEE Antennas Propag. Mag., vol. 49, no. 1, pp. 68-81, Feb. 2007.

[23] M. D. Yacoub, "The $\alpha-\mu$ Distribution: A Physical Fading Model for the Stacy Distribution," IEEE Trans. Veh. Technol., vol. 56, no. 1, pp. 27-34, Jan. 2007.

[24] S. Arzykulov et al., "Hardware- and Interference-Limited Cognitive IoT Relaying NOMA Networks With Imperfect SIC Over Generalized NonHomogeneous Fading Channels," IEEE Access, vol. 8, pp. 72942-72956, 2020.

[25] S. Stefania et al., LTE-The UMTS Long Term Evolution: From Theory to Practice. 2nd ed. New York, NY, USA: Wiley, 2011.

[26] V. S. Adamchik and O. I. Marichev, "The algorithm for calculating integrals of hypergeometric type functions and its realization in REDUCE system," International Symposium on Symbolic and Algebraic Computation (ISSAC), Tokyo, Japan, pp. 212-224, Aug. 1990. 